Revista de Psicología Vol. 37 (1), 2019 (ISSN 0254-9247)

\title{
Formación de Recuerdos Flashbulb en población colombiana
}

\author{
William Tamayo-Agudelo ${ }^{1}$, Julián Cárdenas ${ }^{2}$, Liliana Chaves Castaño ${ }^{3}$ \\ Centro de Habilidades Cognitivas (CHC) - Universidad Cooperativa de \\ Colombia ${ }^{1}$, Universidad de Antioquia, Colombia ${ }^{2,3}$
}

La presente investigación tuvo como objetivo estudiar la formación de los Recuerdos Flashbulb (FBM) en una muestra de población colombiana. Se denomina FBM al tipo de recuerdo autobiográfico que recoge información acerca del contexto de recepción de noticias públicas impactantes. Trescientas ochenta personas (171 hombres y 209 mujeres) entre los 20 y los 89 años respondieron un cuestionario de FBM entre los 4 y 19 días después de publicada una noticia que conmocionó a la población mundial. Se realizaron análisis factoriales para cada grupo de ítems del cuestionario, análisis descriptivos y ecuaciones estructurales. Cuatro modelos de formación fueron probados. Dos modelos, el de Finkenauer et al. (1998) y Er (2003), explicaron la formación de la FBM por un camino indirecto que involucra la memoria del evento como predictor de la FBM. Sin embargo, el modelo de Finkenauer et al. (1998), obtuvo índices de ajuste general más adecuados. Los modelos planteados por Brown y Kulik (1977) y Conway et al. (1994) no predijeron la formación de la FBM en una noticia de interés sobre terrorismo internacional.

Palabras clave: recuerdos flashbulb, modelos de ecuaciones estructurales, modelos teóricos, terrorismo

\section{Flashbulb Memories in a Colombian Sample}

The present research examines the formation of flashbulb memories in a sample of Colombians. Flashbulb Memories (FBM) are autobiographical memories related to the context of receiving shocking public news. A sample of 380 respondents (171 men and 209 women) aged from 20 to 89 years old $(M=37.05$; $S D=13.36)$ conducted a test between $4-19$ days

1 Magíster en Psicología y profesor instructor de la Universidad Cooperativa de ColombiaMedellín. Dirección postal: Carrera 47 Número 37 Sur 18, Envigado, Antioquia (Colombia). Contacto: william.tamayoa@campusucc.edu.co

2 Doctor en Sociología y profesor de tiempo completo del Departamento de Sociología de la Universidad de Antioquia e investigador de Freie Universität Berlin, Alemania. Dirección postal: calle 70 No. 52 - 21 - Apartado aéreo 1226. Contacto: julian.cardenash@gmail.com

3 Magíster en Psicología y vicedecana de la Facultad de Ciencias Sociales Humanas de la Universidad de Antioquia. Dirección postal: calle 70 No. 52 - 21 - Apartado aéreo 1226. Contacto: liliana.chaves@udea.edu.co 
after worldwide breaking news. Factor analysis, descriptive analysis and structural equation models were applied. Four models described by the literature on flashbulb memories were tested. Results show that models proposed by Finkenauer et al. (1998) and Er (2003) predict the formation of flashbulb memories through an indirect path. The models proposed by Brown and Kulik (1977) and Conway et al. (1994) do not predict the formation of flashbulb memories in news related to international terrorism. However, goodness of fit indexes were better for Brown and Kulik's model than for the other three models.

Keywords: Flashbulb memory, Structural Equation Models, theoretical models, terrorism

\section{Formação de Lembranças Flashbulb na populaçáo colombiana}

Esta pesquisa teve como objetivo estudar a formaçáo das Lembranças Flashbulb (FBM) em uma amostra de população colombiana. Chama-se FBM ao tipo de lembranças autobiográficas com informação sobre o contexto de recepção de notícias públicas impactantes. Trezentas e oitenta pessoas ( 171 homens e 209 mulheres) entre 20 e 89 anos responderam um inquérito FBM entre 4 e 19 dias depois da publicação de uma notícia perturbante a nível mundial. Fizeram-se análises fatoriais para cada grupo de itens do inquérito, análises descritivos e equaçóes estruturais. Testaram-se quatro modelos de formação. Dois modelos, o de Finkenauer et al. (1998) e o Er (2003), explicaram a formaçáo da FBM de maneira indireta, envolvendo a memória do evento como preditor da FBM. Porém, o modelo Finkenauer et al. (1998) obteve índices de ajuste geral mais apropriados. Os modelos de Brown e Kulik (1977) e Conway et al. (1994) não predisseram a formação da FBM em uma notícia de interesse sobre terrorismo internacional.

Palavras-chave: memórias Flashbulb, modelos de equaçôes estruturais, modelos teóricos, terrorismo

\section{Formation de Souvenirs Flashbulb dans la population colombienne}

La présente recherche a pour but d'étudier la formation des Souvenirs Flashbulb (FBM) sur un échantillon de population colombienne. On appelle FBM le type de souvenir autobiographique qui recueille des informations sur le contexte de réception d'informations publiques imposantes. Trois-cent quatre-vingts personnes de 20 à 89 ans (171 hommes et 209 femmes), ont répondu à un questionnaire de FBM, de 4 à 19 jours après la publication d'informations qui ont commotionné la population mondiale. Des analyses factorielles, des analyses descriptives et des équations structurelles ont été effectuées pour chaque groupe d'articles du questionnaire. Quatre modèles de formation ont été testés. Deux modèles, celui de Finkenauer et al. (1998) et Er (2003), ont expliqué la formation de la FBM par un chemin indirect qui implique la mémoire de l'événement comme prédicteur de la FBM. Toutefois, le modèle de Finkenauer et al. (1998), a obtenu des taux d'ajustement général plus adéquats. Les modèles proposés par Brown et Kulik (1977) et Conway et al. (1994), n'ont pas prédit la formation de la FBM dans des informations d'intérêt sur le terrorisme international.

Mots-clés: souvenir flashbulb, modèles d'équations structurelles, modèles théoriques, terrorisme 
En los primeros días de enero del año 2015, un comando armado ingresó a la sala de redacción del semanario Charlie Hebdo, ejecutando al editor y a un grupo de periodistas y caricaturistas en lo que fue considerado uno de los ataques más sangrientos ocurrido en las últimas décadas en Paris. Desde el primer momento las sospechas recayeron en grupos de radicales islámicos quienes años atrás habían amenazado a los integrantes del equipo editorial a causa de la publicación de caricaturas satíricas del profeta Mahoma. Poco tiempo después, el grupo Al Qaeda confirmó la autoría del ataque asegurando que habían cumplido con la voluntad de su jefe más reconocido: Osama bin Laden (Joscelyn, 2015). Para muchas personas, este nombre era totalmente desconocido hasta que, en la primera década del siglo XXI, Al Qaeda llevara a cabo uno de los ataques más mediáticos y sangrientos: los atentados al World Trade Center en Nueva York. Antes de este hecho, el nombre de Osama bin Laden era solo reconocido por un reducido grupo de conocedores de la intricada red de poder que se tejió bajo la lumbre de la guerra fría en el Oriente Medio (ver Forigua-Rojas, 2010). Posterior al 11 de septiembre (11-S), la imagen de bin Laden, inundaría las pantallas y las páginas de muchos periódicos y revistas al convertirse en el objetivo de guerra más buscado del mundo. La cacería terminaría en el año 2011 cuando bin Laden fue reportado como dado de baja en una operación realizada por un comando especializado del ejército americano en Pakistán. No obstante, al parecer este hombre continuó siendo un referente para las acciones de Al Qaeda, y eso quedó demostrado con la reivindicación que este grupo realizó luego de la masacre de Charlie Hebdo.

Para justificar el hecho de que la muerte de Osama bin Laden pueda ser considerada como un evento de carácter internacional con repercusiones de variada índole para nuestro continente, pueden aducirse varios aspectos. En primer lugar, su figura, luego del 11-S, fue proyectada como la encarnación del terrorista internacional, sus mensajes recibían cubrimiento periodístico internacional y sus constantes amenazas generaban reacciones inmediatas. En segundo lugar, en una época de sofisticación armamentística, dirigió un ataque a la nación más poderosa del mundo sin utilizar un gran ejército ni armas 
de destrucción masiva. Por último, sus acciones desencadenaron una respuesta militar fuerte y prolongada: la guerra contra el terrorismo decretada por George. W. Bush, que tuvo como consecuencia inmediata el desarrollo de la operación Libertad Duradera (Enduring Freedom).

En Latinoamérica, Colombia respaldó de inmediato la doctrina norteamericana luego del 11-S (Torres Vásquez, 2015). El largo conflicto interno colombiano coadyuvó a que el gobierno de turno se suscribiera a la guerra contra el terrorismo, y los grupos armados del país comenzaron a ser reconocidos como parte del terrorismo internacional (Torres Vásquez, 2015). En esa medida, los atentados del 11-S, la figura de Osama bin Laden y los grupos armados irregulares de Colombia quedaron ligados.

La muerte de Osama bin Laden recibió cobertura mediática internacional. Los noticieros colombianos no fueron la excepción. Incluso noticias relacionadas con él continúan apareciendo en las principales revistas (Revista Semana, 2015). Es por eso por lo que un hecho público de esta dimensión fue estudiado como fenómeno cognitivo y social, a través de la formación de recuerdos personales relacionados con la recepción de la noticia del abatimiento de bin Laden en población colombiana. Este tipo de investigaciones son reconocidas internacionalmente como estudios de Recuerdos Flashbulb (FBM, por sus siglas en inglés) y generalmente están relacionados con hechos impactantes; no obstante, se ha demostrado que hechos esperables, es decir que generan niveles de sorpresa bajos, así como hechos positivos, también pueden ser elicitadores de FBM (Demiray \& Freund, 2015; Lanciano, Curci \& Soleti, 2013; Morse, Woodward \& Zweigenhaft, 1993; Tinti Schmidt, Sotgiu, Testa \& Curci, 2009). En el caso estudiado, podría asumirse que el hecho era esperable, dada la larga persecución internacional a Osama bin Laden. 


\section{Los recuerdos Flashbulb o Recuerdos -Destello}

En 1977, Brown y Kulik se preguntaron por las razones que subyacían al hecho de que recuerdos acerca de detalles que rodearon el recibimiento de una noticia pública impactante fuesen tan vívidos y claros. A este tipo de recuerdos los denominaron Recuerdos Flashbulb (FBM). La característica básica de estos recuerdos es su énfasis en aspectos periféricos como el lugar en donde se estaba cuando se recibió la noticia, la actividad que se realizaba, el medio de información, la respuestas emocionales de las personas que estaban presentes, emociones experimentadas, consecuencias e información idiosindrásica (Edery-Halpern \& Nachson, 2004). La vividez y claridad de estos recuerdos era tan evidente (Brown $\&$ Kulik, 1977) que inicialmente se consideró que habría un mecanismo neurofisiológico (Livingston, 1967) encargado de fijar esta información de manera tan detallada. Con base en ello, Brown y Kulik (1977) plantearon el primer modelo tentativo que explicaba la formación de las FBM. Tres modelos más han sido planteados (Conway et al., 1994; Er, 2003; Finkenauer, et al., 1998). Los cuatro modelos coinciden en que para la formación de una FBM deben confluir aspectos cognitivos, emocionales y sociales. Algunas investigaciones (Er, 2003; Lanciano, Curci \& Semin, 2010) muestran que diferencias en la evaluación cognitiva del evento, los niveles de sorpresa desencadenado por este, así como el grado de implicación de las personas, pueden ser determinantes en su formación.

De acuerdo con el primer modelo (Brown \& Kulik, 1977), los dos principales factores que contribuyen a la formación de las FBM son la reacción de sorpresa en el momento de recibir la noticia y la evaluación de la importancia/consecuencia de dicho evento (Curci \& Luminet, 2009). Brown y Kulik (1977) propusieron que la sorpresa generada por un evento inesperado precede a la evaluación de dicho suceso en términos de su importancia/consecuencia y provoca un alto grado de activación (arousal) emocional (Tinti, Schmidt, Sotgiu, Testa \& Curci, 2009). Además, sostenían que la importancia/consecuencia atribuida al hecho junto con una intensa activación emocional, favorecían el que se hablara más acerca de ello (Tinti et al., 2009). Este modelo se reconoce 
actualmente como Modelo Fotográfico. El segundo modelo (Conway et al., 1994), se denomina Modelo Comprensivo. Postula que dos variables latentes tendrían efectos directos sobre la formación de la FBM: emocionalidad y recuento o repetición. Otras dos duplas de variables, Conocimiento/Interés e Importancia/Consecuencias tendrían efectos indirectos. En este modelo, el conocimiento previo asume el papel de organizador de la memoria, y apoyado en la teoría de los Modelos de Ecuaciones Estructurales, detalla las diferencias entre efectos directos e indirectos en la formación de una FBM.

El tercer modelo, propuesto por Finkenauer et al. (1998), postula que el proceso de formación y mantenimiento de las FBM se daría por dos vías. Una directa, que va de la respuesta de sorpresa hacia la evaluación cognitiva de la novedad y de esta a la FBM, y una indirecta que señala que la formación de la FBM comienza en la evaluación cognitiva de la importancia y las consecuencias personales que se desencadenan en el momento en que se recibe una noticia impactante. Esto afectaría las respuestas emocionales de sentimientos negativos, lo que a su vez llevaría a la aparición de conductas sociales de intercambio o recuento de los hechos (rehearsal), con lo cual se fortalecería la huella de memoria del evento (ME) como tal. En ese sentido, para esta vía, el predictor directo será la memoria semántica, representada en la ME.

En el cuarto modelo, Er (2003), investigó la formación de FBM en personas que vivieron el terremoto de Turquía en 1999 (grupo de víctimas o personas con alta implicación y personas que solamente escucharon acerca de él a través de los medios (grupo de no víctimas o baja implicación). Este autor sugiere que los recuerdos del grupo de mayor implicación, son diferentes de los de aquellos que no vivieron la experiencia de forma directa. Por lo tanto, dos modelos estructurales son necesarios para entender la formación de la FBM (Er, 2003). La diferencia más importante entre los modelos anteriores y el nuevo modelo de Er (2003), fue encontrar que en los sujetos que habían vivido el evento directamente, la $\mathrm{ME}$ no se diferenciaba de los recuerdos de recepción del contexto. Esto es, la FBM es equivalente a la ME (Er, 2003). Asimismo, del análisis estructural surgió un camino directo 
de la evaluación de la importancia/consecuencia hacia la FBM, hecho que contradice los postulados de los modelos anteriores, donde la relación es indirecta. Entre tanto, el modelo para el grupo de recepción indirecta se asemeja al planteado por Finkenauer et al. (1998).

Aunque los modelos plantean relaciones diferentes entre las variables, se pueden encontrar algunas regularidades (Luminet \& Curci, 2009). En la formación de una FBM siempre se encuentra implicada la reacción de sorpresa; hay una evaluación cognitiva de su importancia/consecuencia para el individuo; la intensidad de las reacciones emocionales juega un papel fundamental y por último el aspecto de las conductas de recuento abiertas (conversaciones) y encubiertas (pensamientos) influye directa o indirectamente en la FBM. Nuestro objetivo principal fue estudiar la formación de Recuerdos Flashbulb (FBM) en población colombiana a través de una noticia de trascendencia mundial: la muerte de Osama bin Laden. Estudios previos (Finkenauer et al., 1998; Luminet \& Curci, 2009) han mostrado que el modelo de Finkenauer et al. (1998), se ajusta mejor a la explicación de la formación de una FBM. Nuestra hipótesis inicial es que este modelo explicará la formación de una FBM en población colombiana. Además, dado que $\operatorname{Er}$ (2003) propuso un modelo para no implicados (en este caso puede considerarse que los participantes cumplen esta condición), este modelo debe tener unos índices aceptables para explicar la formación de dichos recuerdos.

\section{Método}

\section{Participantes}

Una muestra no probabilística de trescientas ochenta personas entre 20 y 89 años $(M=37 ; D E=13.4)$, conformados por 209 mujeres y 171 hombres residentes en la ciudad de Medellín, Colombia, respondieron un cuestionario de FBM acerca de la muerte de Osama bin Laden, entre los 4 y 19 días posteriores al evento. El porcentaje más alto de participantes era soltero $(44.7 \%)$ y con una formación académica media (27.9\% formación técnica o tecnológica; $26.3 \%$ secundaria completa). 


\section{Medición}

El cuestionario utilizado fue descrito por Curci \& Luminet (2006), Luminet \& Curci (2009) y Luminet et al. (2004). Originalmente se utilizó para la evaluación de los recuerdos del contexto de recepción de la noticia de los ataques a las Torres Gemelas, así como de los determinantes psicológicos y contextuales involucrados en su formación. Para los propósitos de esta investigación, se adaptó el cuestionario siguiendo la prueba descrita por Luminet \& Curci (2009) debido a que recoge todos los determinantes que inciden en la formación de FBM, y las propiedades psicométricas reportadas fueron adecuadas. El cuestionario se organiza en nueve categorías. La descripción del instrumento puede encontrarse en la tabla 1 . Sin embargo, una descripción extensa y detallada de las propiedades psicométricas, puede encontrarse en Tamayo-Agudelo, Chaves Castaño \& Cárdenas (2017).

\section{Tabla 1}

Organización del instrumento

\begin{tabular}{|c|c|c|}
\hline Categoría & Descripción del ítem & Calificación \\
\hline \multirow{5}{*}{$\begin{array}{l}\text { Memoria del } \\
\text { contexto de } \\
\text { recepción } \\
(\text { FBM) }\end{array}$} & Hora en la que recibieron la noticia & \multirow{5}{*}{$\begin{array}{l}\text { Puntuación de } 0 \text { a } 5 \\
0=\text { ausencia de } \\
\text { respuesta o muy } \\
\text { general (p.e. mañana, } \\
\text { tarde) } \\
1=\text { por cada detalle } \\
\text { expresado claramente } \\
\text { (máx. 5) }\end{array}$} \\
\hline & $\begin{array}{l}\text { Lugar en que se encontraban cuando se } \\
\text { enteraron }\end{array}$ & \\
\hline & $\begin{array}{l}\text { Fuente de la información (periódicos, } \\
\text { noticieros, otra persona, etc.) }\end{array}$ & \\
\hline & $\begin{array}{l}\text { Personas presentes en el momento de la } \\
\text { recepción de la noticia }\end{array}$ & \\
\hline & Actividad que estaban realizando & \\
\hline \multirow{6}{*}{$\begin{array}{l}\text { Memoria del } \\
\text { evento }\end{array}$} & $\begin{array}{l}\text { Fecha exacta de la muerte de Osama bin } \\
\text { Laden }\end{array}$ & \multirow{6}{*}{$\begin{array}{l}\text { Puntuación de } 0 \text { a } 2 \\
0=\text { respuesta incorrecta } \\
1=\text { respuesta } \\
\text { parcialmente correcta } \\
2=\text { respuesta correcta }\end{array}$} \\
\hline & Qué día de la semana fue la muerte & \\
\hline & Hora de la muerte & \\
\hline & Número de personas que murieron con él & \\
\hline & $\begin{array}{l}\text { Nombre del lugar donde murió (país y/o } \\
\text { ciudad) }\end{array}$ & \\
\hline & Qué sucedió con el cadáver & \\
\hline
\end{tabular}




\begin{tabular}{|c|c|c|}
\hline Categoría & Descripción del ítem & Calificación \\
\hline \multirow{3}{*}{$\begin{array}{l}\text { Evaluación de } \\
\text { la novedad }\end{array}$} & Qué tan común fue el evento (Inverso) & Puntuación de 1 a 7 \\
\hline & Qué tan significativo fue el evento & $0=$ nada \\
\hline & & $7=$ mucho \\
\hline \multirow{3}{*}{$\begin{array}{l}\text { Evaluación de } \\
\text { la sorpresa }\end{array}$} & Qué tan sorprendente fue el evento & Puntuación de 1 a 7 \\
\hline & Qué tan esperable fue el evento (Inverso) & $0=$ nada \\
\hline & & $7=$ mucho \\
\hline \multirow{6}{*}{$\begin{array}{l}\text { Evaluación de } \\
\text { la importancia/ } \\
\text { consecuencia }\end{array}$} & $\begin{array}{l}\text { Qué importancia tuvo la muerte de Osama } \\
\text { bin Laden internacionalmente }\end{array}$ & \multirow{6}{*}{$\begin{array}{l}\text { Puntuación de } 1 \text { a } 7 \\
0=\text { nada } \\
7=\text { mucho }\end{array}$} \\
\hline & $\begin{array}{l}\text { Qué importancia tuvo la muerte de Osama } \\
\text { bin Laden para el país }\end{array}$ & \\
\hline & Qué importancia tuvo la muerte de Osama & \\
\hline & bin Laden para su familia y amigos & \\
\hline & $\begin{array}{l}\text { Qué importancia tuvo la muerte de Osama } \\
\text { bin Laden para usted }\end{array}$ & \\
\hline & $\begin{array}{l}\text { Qué consecuencias tuvo la muerte de Osama } \\
\text { bin Laden para usted }\end{array}$ & \\
\hline \multirow{3}{*}{$\begin{array}{l}\text { Intensidad } \\
\text { de emociones } \\
\text { asociadas al } \\
\text { evento }\end{array}$} & Qué tanto lo alteró la noticia de la muerte & \multirow{3}{*}{$\begin{array}{l}\text { Puntuación de } 1 \text { a } 7 \\
0=\text { nada } \\
7=\text { mucho }\end{array}$} \\
\hline & Qué tan conmovido se sintió & \\
\hline & Qué tanto le impactó & \\
\hline
\end{tabular}

Qué tanto les agradan los Estados Unidos de

América como país

Qué tanto les agradan los ciudadanos de los

Estados Unidos

Puntuación de 1 a 7

Actitudes

Qué tanto apoyan la búsqueda de un

$0=$ nada

fugitivo en un país extranjero por parte del $\quad 7=$ mucho

país afectado

Qué tanto apoyan el asesinato de fugitivos en

países extranjeros por parte del país afectado

Con qué frecuencia tuvieron pensamientos, recuerdos o imágenes relacionadas con la muerte de bin Laden en los últimos 3 días

Puntuación de 1 a 5

Repetición

Cuántas veces hablaron de la muerte de

$1=$ ninguna

Osama en los últimos 3 días

7 = quince $o$ más veces

En los últimos 3 días, cuántas veces siguieron

el caso en periódicos o noticieros 


\begin{tabular}{|c|c|c|}
\hline Categoría & Descripción del ítem & Calificación \\
\hline \multirow{5}{*}{$\begin{array}{l}\text { Conoci- } \\
\text { miento } \\
\text { previo }\end{array}$} & $\begin{array}{l}\text { Cuál era la nacionalidad de Osama bin } \\
\text { Laden } \\
\text { En qué país se sospechaba que estaba } \\
\text { escondido }\end{array}$ & $\begin{array}{l}\text { Puntuación de } 0 \text { a } 1 \\
0=\text { respuesta incorrecta } \\
1=\text { respuesta correcta }\end{array}$ \\
\hline & Por qué razón era conocido a nivel mundial & Puntuación de 0 a 3 \\
\hline & & $0=$ respuesta incorrecta \\
\hline & & $1=$ respuesta correcta \\
\hline & Nombre del grupo terrorista que lideraba & $\begin{array}{l}3=\text { respuestas } \\
\text { que adicionaban } \\
\text { información relevante } \\
\text { para complementar la } \\
\text { respuesta }\end{array}$ \\
\hline
\end{tabular}

Nota. Para evitar el efecto techo, los participantes acompañaron hasta con cinco detalles específicos los recuerdos de la hora, el lugar, la fuente de información, con quién se encontraban y lo que hacían cuando recibieron la noticia. Los detalles fueron calificados siguiendo cuatro criterios básicos, dos tomados de Finkenauer et al. (1998), y otros dos propuestos por los investigadores: (1) el detalle responde a un aspecto cambiante del ambiente que difícilmente puede ser inferido por la familiaridad del sujeto con el espacio (v.g., el perro estaba en frente echado sobre una poltrona $=1$; mi mamá me traía un jugo $=1$ ); (2) el recuerdo corresponde a la posición espacial del participante en relación al ambiente en ese momento específico (estaba sentada en la cabecera de la mesa del comedor=1); (3) el detalle responde a aspectos del entorno que han sido considerados como información periférica relacionada con la recepción de la noticia y que directamente no se preguntaron (descripción de la ropa que se llevaba puesta, estado del clima ese día); (4) expresiones o pensamientos específicos que vinieron a la mente justo cuando se escuchó la noticia (v.g., pensé: ¡casi que no!= 1; pensé en lo malo que era= 0 ). La información de las MFB de la muerte de Osama bin Laden y los cinco detalles se sumaron, obteniéndose un índice de MFB.

\section{Procedimiento}

Los participantes leyeron y firmaron un detallado consentimiento informado para investigaciones de mínimo riesgo en el cual se les brindaba la definición de las FBM, los objetivos principales del proyecto y la advertencia de que podían retirarse de la investigación en cualquier momento sin dar cuenta de las motivaciones de su decisión. El diligenciamiento del cuestionario tuvo una duración aproximada de 30 minutos. 


\section{Análisis de datos}

Tres pasos se siguieron para realizar el análisis de los datos. En primer lugar, se realizaron los análisis factoriales (AF) a cada una de las dimensiones de la escala. En segundo lugar, se obtuvieron los estadísticos descriptivos; y por último se probaron los diversos modelos propuestos por la teoría.

\section{Resultados}

\section{Análisis factoriales del instrumento}

Se realizaron análisis individuales para los grupos de ítems, asumiendo que cada uno de ellos puede explicarse por una única dimensión que explica la mayor parte de la varianza común. Esta solución es útil para los propósitos de esta investigación en la medida que es necesario, por un lado, identificar las variables latentes que subyacen a cada grupo de ítems, y por el otro, crear una nueva medida que represente cada variable en función de las variables observadas (Luminet et al., 2004). La determinación de la unidimensionalidad se realizó por medio del análisis paralelo (Timmerman \& Lorenzo-Seva, 2011), y para los AF se utilizó el método de Análisis Factorial de Rango Mínimo (MRFA). Los análisis se realizaron sobre matrices no lineales (Ferrando \& Lorenzo-Seva, 2014) y se determinó la varianza común explicada y la confiabilidad para cada factor (ver tabla 2).

Como puede observarse en la tabla 2, la prueba tuvo un buen comportamiento, con medidas de confiabilidad alpha de Cronbach en un rango de .64 a .80 . El análisis paralelo confirmó el supuesto de unidimensionalidad para cada grupo de ítems, mientras que las varianzas comunes explicadas fueron altas (rango 70.68\% - 100\%), lo que indicaría que los ítems o variables observables representaban de una manera adecuada el factor latente (Tamayo-Agudelo et al., 2017). 


\section{Tabla 2}

Varianza y Confiabilidad de los Factores del Instrumento

\begin{tabular}{lcc}
\hline Factor & \% varianza común explicada & $\alpha$ \\
\hline FBM muerte de OBL & 78.37 & .79 \\
Memoria del Evento & 70.68 & .69 \\
Evaluación Novedad/Sorpresa & 75.19 & .64 \\
Evaluación Importancia/Consecuencia & 74.72 & .73 \\
Emocionalidad & 99 & .73 \\
Actitudes & 75.06 & .80 \\
Repetición (Rehearsal) & 100 & .80 \\
Conocimiento previo & 89.86 & .64 \\
\hline
\end{tabular}

FBM: Memoria del contexto de recepción; OBL: Osama bin Laden

\section{Descripción de las variables}

FBM de la muerte de Osama bin Laden. La hora en que se recibió la noticia tuvo una media de $.93(D E=.26)$. El lugar en que se encontraban los participantes tuvo una media de $.95(D E=.22)$. Los participantes también recordaban el medio de información $(M=.96$; $D E=.195)$, las personas que acompañaban al participante $(M=.92$; $D E=.27)$ y la actividad que realizaban $(M=.94 ; D E=.24)$.

Variables predictoras. Las variables relacionadas con la Memoria del Evento tienen que ver con el día de la semana en que murió Osama bin Laden $(M=.03 ; D E=.18)$; la fecha exacta $(M=.96 ; D E=.96)$; hora de la muerte $(M=.24 ; D E=.64)$; número de personas que murieron $(M=.66 ; D E=.71)$; lugar de la muerte $(M=.50 ; D E=.54)$ y hechos relacionados con el destino del cadáver $(M=.74 ; D E=.499)$. Estos datos permiten observar que la mayoría de los participantes tienden a recordar la fecha de la muerte de Osama bin Laden, el número de personas que murieron en el operativo y el destino de cadáver.

En la variable Novedad, los resultados permiten observar que a los participantes el evento les pareció novedoso $(M=5.79 ; D E=1.91)$ y significativo $(M=5.87 ; D E=1.814)$. 
En contraste, la variable Sorpresa es descrita por los participantes en un nivel medio $(M=4.21 ; D E=2.32)$, lo que indica que la muerte de Osama bin Laden era esperable.

La importancia/consecuencia mostró que los participantes le otorgan al evento una alta relevancia internacional $(M=6.56$; $D E=1.04)$. La importancia para Colombia $(M=4.22 ; D E=1.99)$ es media, al igual que para la familia y los amigos de los participantes $(M=3,09 ; D E=2,22)$. La relevancia personal de la muerte de bin Laden tiene una media de $3.06(D E=2.28)$. Los participantes no le otorgan mucha consecuencialidad al evento $(M=1.90 ; D E=1.73)$.

En la variable que explora el Estado Emocional de las personas al recibir la noticia se encontró que los participantes se alteraron poco $(M=1.86 ; D E=1.66)$, mientras que los niveles de conmoción $(M=2.04 ; D E=1.72)$ y de impacto fueron más altos $(M=3.94$; $D E=2.31)$.

La Actitud mostró que los participantes sienten agrado por los Estados Unidos $(M=4.39 ; D E=2.12)$ y por sus ciudadanos $(M=4.33$; $D E=1.94)$. Asimismo, los participantes apoyan la búsqueda de fugitivos en otro país $(M=4.82 ; D E=2.24)$ y su asesinato $(M=4.11$; $D E=2.36$ ).

La variable Repetición mostró que después de un rango entre 4 y 19 días después de la noticia, los participantes recordaron, pensaron o tuvieron imágenes asociadas a la muerte de bin Laden en un bajo promedio $(M=1.88 ; D E=1.18)$. Hablar del evento y hacer un recuento del mismo se ubicó con una media de 2,14 $(D E=1.22)$. El seguimiento de las noticias también tuvo una distribución normal $(M=2.09 ; D E=1.28)$.

Los Conocimientos Previos sobre los antecedentes de Osama bin Laden que reportaron los participantes reflejan baja información sobre su nacionalidad $(M=.25 ; D E=.48)$, lugar donde se sospechaba que estaba escondido $(M=.82 ; D E=.88)$, evento por el cual era conocido a nivel mundial $(M=.92 ; D E=.55)$ y grupo terrorista del que bin Laden era líder $(M=.77 ; D E=.45)$. 


\section{Comparación de Modelos de Ecuaciones Estructurales}

Los Modelos de Ecuaciones Estructurales (MEE) comprenden un conjunto de modelos de análisis que permiten establecer las relaciones y efectos entre variables utilizando medidas múltiples (Pérez, Medrano \& Sánchez Rosas, 2013; Ruiz, Pardo \& San Martin, 2010). Una ventaja de los MEE frente a otro tipo de análisis es que permiten probar hipótesis de causalidad respecto a las variables planteadas en un modelo. Para este caso particular se sigue el método de máxima verosimilitud, se prueban modelos ya planteados teóricamente y se comparan los índices de ajuste que en la literatura son los más aceptados. La prueba de chi-cuadrado, GFI, RMSEA, AGFI, TLI y la chi-cuadrado normada (valor del chi-cuadrado entre los grados de libertad) son las medidas de ajuste comparadas en los diversos modelos.

En la tabla 3 se muestran los resultados de las medidas de ajuste de los diferentes modelos. Nuestros resultados muestran que el modelo con índices menos satisfactorios es el denominado Modelo Comprensivo (Conway et al., 1994). Aunque el GFI y el AGFI alcanzan valores aceptables los restantes índices no se ajustan a lo esperado.

\section{Tabla 3}

Indices de Bondad de Ajuste para la modelización de las FBM de la muerte de Osama bin Laden

\begin{tabular}{|c|c|c|c|c|c|c|c|}
\hline & $\chi^{2}$ & $\mathrm{gl}$ & $\chi^{2} / \mathrm{gl}$ & GFI & RMSEA & AGFI & TLI \\
\hline $\begin{array}{l}\text { Modelo de Brown \& } \\
\text { Kulik (1977) }\end{array}$ & 6.167 & 6 & 1.02 & .995 & .009 & .981 & .997 \\
\hline $\begin{array}{l}\text { Modelo de Conway } \\
\text { et al. (1994) }\end{array}$ & 29.141 & 3 & 9.71 & .971 & .152 & .854 & .265 \\
\hline $\begin{array}{l}\text { Modelo de Finkenauer } \\
\text { et al. (1998) }\end{array}$ & 44.217 & 17 & 2.60 & .972 & .065 & .941 & .797 \\
\hline Modelo de Er (2003) & 32.694 & 11 & 2.97 & .976 & .072 & .940 & .789 \\
\hline
\end{tabular}




\section{Resultados de los caminos significativos para la formación de la FBM de la muerte de Osama bin Laden}

Los cuatro modelos de formación de las FBM de la muerte de Osama bin Laden fueron probados comenzando por el Modelo Fotográfico (Brown \& Kulik, 1977). En nuestros datos (ver Figura 1), los caminos propuestos por este modelo fueron significativos solo entre las variables predictoras, pero no hay relación entre estas y la FBM. Es llamativo que hay una relación negativa entre el índice de sorpresa y el de importancia: a mayor importancia del suceso menor sorpresa. La noticia de la muerte de bin Laden, aunque es altamente importante, es esperable. Este hallazgo no se corresponde con los resultados de Conway et al. (1994) ya que en sus estudios a mayor sorpresa mayor importancia de la noticia.

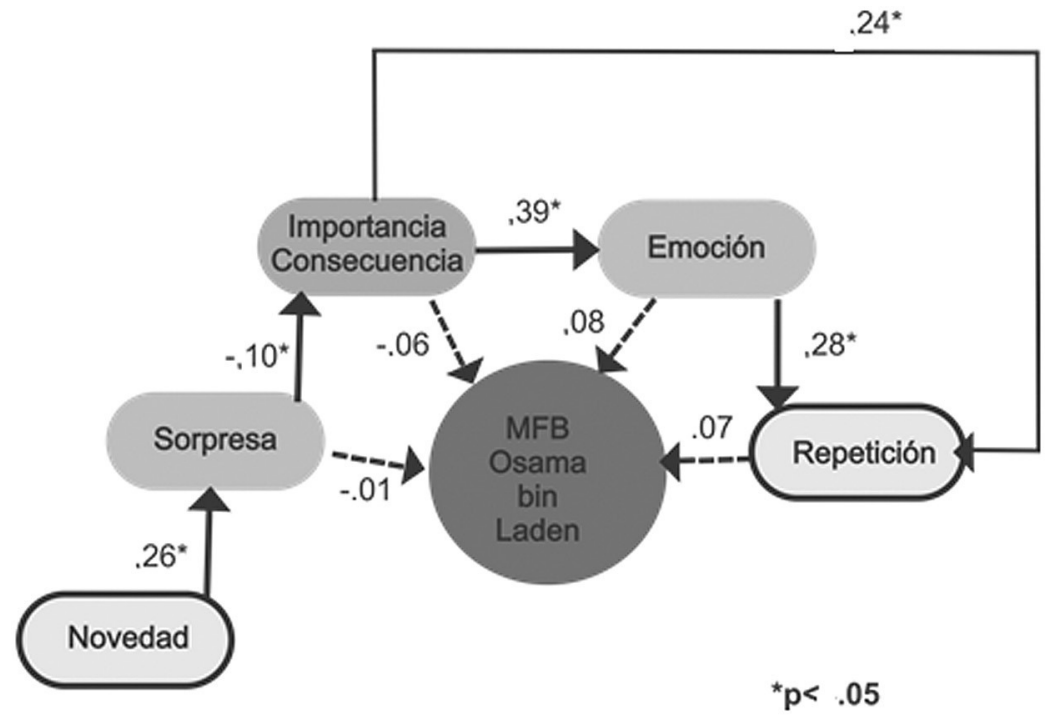

Nota. Los caminos significativos se marcaron con líneas continuas.

Figura 1. Modelo fotográfico propuesto por Brown \& Kulik (1977). 
En cuanto al Modelo Comprensivo de Conway et al., 1994, en la figura 2 se observan los caminos que obtuvieron índices significativos para la formación de la FBM de la muerte de bin Laden. Resalta el hecho de que el conocimiento previo contribuya negativamente sobre la evaluación de la importancia. Esto es, cuanto más sabe la gente acerca de bin Laden, menos importancia le atribuye al evento.

Otro camino predicho por el Modelo Comprensivo, cuyo índice fue significativo en nuestro análisis, es el que conecta el conocimiento con la repetición. Entre tanto, el camino que surge del conocimiento hacia las respuestas emocionales, no lo fue.

En cuanto a los caminos predichos por el modelo y que surgen de la evaluación de la importancia hacia la repetición y las emociones generadas por la noticia, ambos fueron significativos. No obstante, estas dos últimas variables no mostraron tener incidencia en la formación de la FBM de la noticia de la muerte de bin Laden, contradiciendo así lo planteado por Conway et al. (1994).

En cuanto al modelo de Finkenauer et al. (1998), los resultados muestran que la mayoría de vías del camino indirecto postulado por el modelo, fueron significativas. Solo la vía que surge del conocimiento y las actitudes previas hacia la respuesta emocional no lo fue. Esto indica que ni el conocimiento previo ni las actitudes afectaron la respuesta emocional inicial ante la recepción de la noticia.

En cuanto al camino directo que liga la evaluación de la novedad con los niveles de sorpresa y de esta hacia la respuesta emocional, solo la novedad influyó sobre la respuesta de sorpresa sin mostrarse significativa ninguna otra vía; por lo cual este camino no se mostró predictor de la formación de la FBM (ver Figura 3).

Por último, el modelo desarrollado por Er (2004) (ver Figura 4) para grupos de no implicados en los acontecimientos medidos, fue probado. Este modelo es muy semejante al elaborado por Finkenauer et al. (1998). Las diferencias básicas entre estos son: 1) Er no diferencia la evaluación de la novedad y de la sorpresa y 2) a diferencia de los estudios de Conway et al. (1994) y Finkenauer et al. (1998), Er no midió en su estudio original la variable conocimiento previo (Luminet, 2009). 


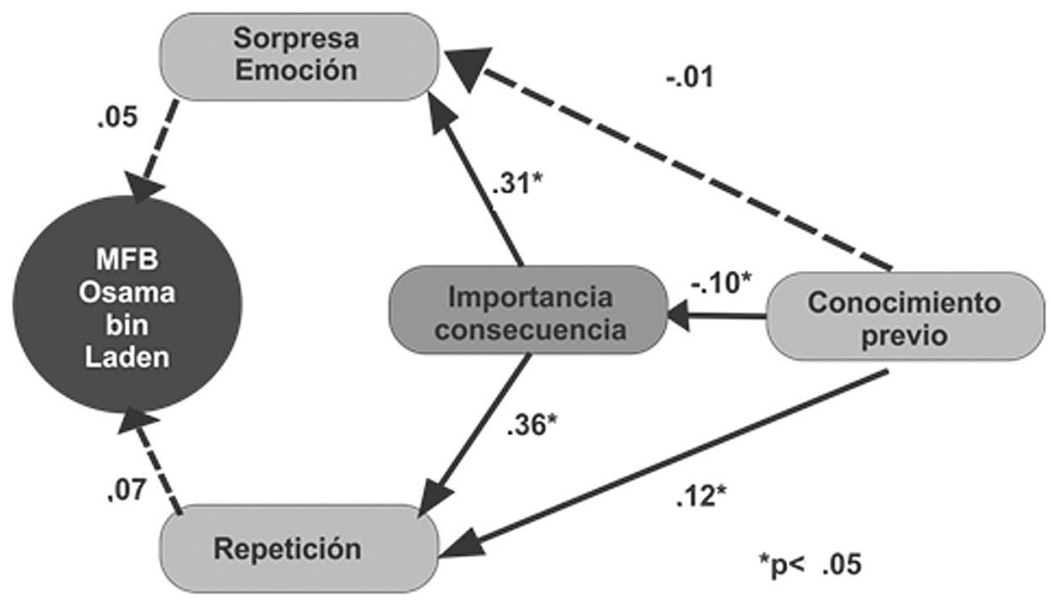

Nota. Los caminos significativos se marcaron con líneas continuas.

Figura 2. Modelo de formación de las FBM propuesto por Conway et al. (1994).

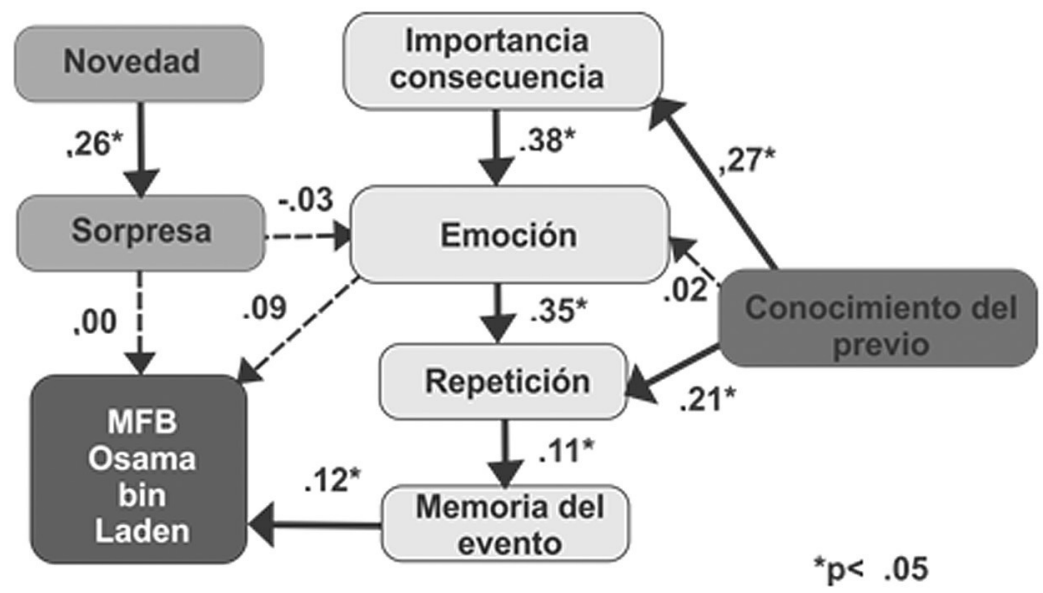

Nota. Los caminos significativos se marcaron con líneas continuas.

Figura 3. Modelo de formación de las FBM propuesto por Finkenauer et al. (1998). 


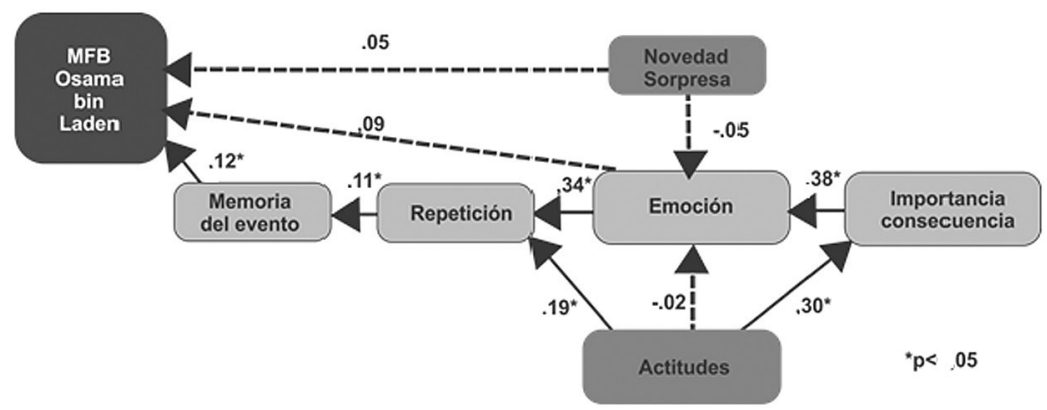

Nota. Los caminos significativos se marcaron con líneas continuas.

Figura 4. Modelo de formación de las FBM propuesto por Er (2003).

Nuestros resultados de la prueba de este modelo son muy similares a los encontrados en la prueba del modelo de Finkenauer et al. (1998). El camino directo de formación que va de la Novedad/sorpresa vía la emoción, no se encontró significativo para la formación de la FBM. El camino indirecto, que implica la evaluación cognitiva de la importancia del evento que activa una respuesta emocional que a su vez lleva a una activa repetición y búsqueda de información sobre el hecho y de allí a una consolidación de la memoria del evento, siendo esta última la predictora directa de la formación de la FBM, fue significativo. Dos de los tres caminos que surgen de la variable predictora Actitudes se hallaron como significativos. Solo el camino que va de las Actitudes a la Emoción no lo fue.

\section{Discusión}

El principal hallazgo de esta investigación es que el modelo de Finkenauer et al. (1998) se ajustó de manera satisfactoria a los datos provenientes de población colombiana relacionados con la formación de la FBM de la muerte de Osama bin Laden, comparado con los modelos restantes, tal y como se planteaba en nuestra hipótesis. 
Los resultados mostraron que el proceso de formación sigue un camino extenso en el cual la emoción experimentada al recibir la noticia está condicionada por la evaluación cognitiva de la importancia atribuida al hecho. El proceso de recuento del hecho lleva a que se forme la ME y esta a su vez es el predictor directo de la FBM. El camino emocional directo para la formación de la FBM, no se mostró significativo.

Este resultado puede ser comprendido desde dos vertientes. En primer lugar, Luminet \& Curci (2009) encontraron que el camino indirecto de este modelo fue el más adecuado para explicar la formación de la FBM del 11-S en no norteamericanos, mientras que, para los norteamericanos, el camino emocional directo fue el más explicativo. Lo que podrían estar indicando estos datos es que tal y como lo postula Er (2003), el proceso de formación de las FBM está determinado por el grado de implicación que se tenga con el evento. En nuestro caso, la noticia de la muerte de Osama bin Laden, tomado como evento elicitador de una FBM, probablemente no desencadenaría respuestas emocionales intensas y, por tanto, los niveles de evaluación de la novedad y los de la sorpresa no alcanzasen el nivel suficiente como para desencadenar una respuesta que formara, por un camino directo, la FBM. Esto mismo cabe pensar para entender la razón por la cual, a diferencia de los hallazgos reportados previamente por Curci \& Luminet (2009) respecto a la formación de la FBM de la muerte del expresidente Miterrand, no se encontró conexión directa entre los estados emocionales y la FBM de la muerte de bin Laden.

En segundo lugar, en un estudio previo, Curci \& Luminet (2009) mostraron que un evento esperable, no sorpresivo, podría también generar una FBM y para explicar su formación, el modelo de Finkenauer et al. (1998) era adecuado. Nuestros resultados muestran que la muerte de bin Laden era esperable, considerando la persecución a la que fue sometido luego de los atentados del 11-S. Esto aportaría nueva evidencia de que la sorpresa no es un factor determinante para la formación de una FBM (Curci, 2005; Curci \& Luminet, 2009; Curci, Luminet, Finkenauer \& Gisle, 2001; Lanziano et al., 2013; Morse et al., 1993; Tinti, et al., 2009). 
Un punto particular que debe discutirse es el papel de aspectos sociales en la formación de FBM. Teóricamente, Bernsten (2009) ha propuesto un modelo en el cual la identidad social es el principal factor para entender las razones subyacentes de fijación de recuerdos personales asociados a hechos sociales. En esa medida, el evento será evaluado como importante en función de la activación de la identidad social del sujeto. Bajo esta condición, la FBM, será una consecuencia directa de las representaciones compartidas grupalmente. En el marco de nuestros resultados, sin embargo, ninguno de los modelos probados analiza específicamente este aspecto. En efecto, de las tres variables que componen el índice de repetición, solo una puede ser considerada abiertamente social. Al preguntar por el número de veces que se habló acerca del evento, implícitamente se asume que el intercambio social es un mecanismo de consolidación del recuerdo y, además, como insinúan algunos autores (Bernsten, 2009; Neisser, 1982) lo transforma. No obstante, es necesario explorar nuevas vías de comprensión tomando como base lo social.

Una representación social puede entenderse como "....un complejo indiferenciado de 'elementos cognitivos' relativos a un objeto social" (Rateau \& Lo Monaco, 2013, p. 25). La teoría de las representaciones sociales sostiene que no todo hecho es susceptible de ser representado socialmente. Para el caso de los hechos estudiados como fuente de FBM, la particularidad de los mismos, y el cubrimiento mediático, los hace más susceptibles de ser representados por una comunidad. En esa medida, la fuente de información se torna un factor determinante en la perspectiva social de abordaje del evento y también en la formación de la FBM (Lanziano, Curci \& Soleti, 2013). Para los casos de estudios de FBM, podría asumirse que la formación de un recuerdo personal asociado a un hecho social implica que exista o se haya formado una representación social que permita una identificación y un intercambio en una comunidad. De acuerdo con ello, es posible especular que los atentados terroristas del 11-S tengan una representación social en la población colombiana, y la figura de Osama bin Laden sea un derivado de esta representación, y, por tanto, esto ayude a entender que, a pesar de la baja sorpresa generada por el suceso, sea posible estudiar su muerte como una fuente de FBM. 
Del mismo lado, la representación social de un objeto se enmarca dentro de una arquitectura del pensamiento social (Flament \& Rouquette, 2003), lo que indica que en sí misma congrega actitudes y opiniones, pero además representa un modo de conocimiento social relacionado con una ideología. Si la representación social de un objeto sirve de base para la formación de una FBM, se deduce que las FBM deben verse afectadas de manera diferencial por los diferentes niveles del pensamiento social. Sin embargo, este hecho no ha sido abordado aun (Tamayo Agudelo, 2012). En todo caso, lo social ha sido resaltado como factor interviniente en la rememoración social (Halbwachs, 1994/2004) y este tipo de recuerdos tiene una fuerte carga de esta variable que debe ser explorada y que debe tener una representación más clara dentro de los modelos.

Continuando con el análisis de las pruebas de los modelos, nuestros resultados muestran que el modelo con mejores índices de bondad de ajuste fue el Modelo Fotográfico (Brown \& Kulik, 1977), aunque las variables predictoras no influyeron significativamente sobre las FBM (ver la figura 1). Vale resaltar que en este modelo la variable que posee el mayor peso es la evaluación cognitiva de la importancia/consecuencia sobre la emoción $(\beta=.39)$, un camino que mostró coeficientes significativos y en general más altos que los demás, en todos los modelos evaluados. Todas las medidas de ajuste de este modelo superaron en adecuación a los otros tres (ver la tabla 3). Estos resultados contradicen los hallazgos de Er (2003), Finkenauer et al. (1998) y Luminet $\&$ Curci (2009) quienes, encontraron en este modelo ajustes pobres para sus datos. En los resultados obtenidos en la presente investigación, los caminos causales propuestos por este modelo como determinantes directos de la FBM, no fueron significativos. Ya Finkenauer et al. (1998) y Luminet (2009), habían señalado la carencia en el Modelo Fotográfico de una base teórica sólida y la indistinción en el manejo de constructos como evaluación de la consecuencia y estados emocionales, constructos que en la investigación en psicología cognitiva de las emociones han sido diferenciados (Fridja, 1986; Fridja, Kuipers \& ter Schure, 1989; Lazarus \& Folkman 1986). Se entiende que para 
Finkenauer et al. (1998) y Luminet (2009), los bajos índices de ajuste de este modelo están explicados por estos vacíos. Recientemente, Luminet (2018) ha decidido no considerar más el Modelo Fotográfico dentro de los análisis teóricos y empíricos de las FBM, puesto que Brown y Kulik no probaron su modelo.

Mirado con detalle, el Modelo Fotográfico se sustenta en el carácter inesperado y sorpresivo del evento. En nuestros datos, la sorpresa no fue alta, y esto se refleja en el modelo. Luminet \& Curci (2009), encontraron que el único camino significativo de causalidad propuesto por este modelo en población norteamericana frente a los atentados del 11-S fue el que conecta la sorpresa con la FBM directamente; mientras que, en el grupo de extranjeros, fue la repetición. Lógicamente los niveles de evaluación y generación de sorpresa despertados por los ataques del 11-S fueron mayores en población americana que en cualquier otro país, por lo que no se extrańa que este camino se mostrase significativo para Luminet \& Curci (2009). Asimismo, esos niveles no necesariamente tenían que ser tan elevados para los no americanos, por lo que la repetición puede ser entendida como la vía determinante para ellos. En la presente investigación, los niveles medios de sorpresa probablemente influyeron en el hecho de que a pesar de que el modelo se ajustase bien a los datos, no predijese la formación de la FBM.

El modelo propuesto por Er (2003) también obtuvo índices aceptables y explicó la formación de la FBM por una vía indirecta. En su estudio original, esta autora propuso dos modelos. Para ella, en el caso de sujetos que han vivenciado los hechos o fueron víctimas, la ME es la misma FBM. Entre tanto, para el modelo de personas no víctimas, la evaluación de la novedad y la sorpresa por un lado y la ME por el otro, son los directos predictores, muy semejante a lo propuesto por Finkenauer et al. (1998). El modelo de Er (2003) para no víctimas, se mostró también adecuado en nuestro caso, aunque con índices inferiores de adecuación comparado con el modelo de Finkenauer et al. (1998). Este hallazgo contradice en parte los resultados de la misma Er (2003), quien reportó índices superiores de ajuste en su modelo superiores a los de Finkenauer et al. (1998). No obstante, Luminet 
(2018) ha levantado una crítica a este resultado basándose en el hecho de que Er mejoró el ajuste global del modelo siguiendo modificaciones a algunos índices. Con todo, el modelo propuesto por Er es una buena alternativa para futuros estudios de formación de FBM. No obstante, sería mejor tomarlo en cuenta cuando los hechos estudiados involucren población víctima, pues la racionalidad del modelo y su axiomática se estableció partiendo de la implicación directa de las personas evaluadas.

El modelo con menor adecuación a los datos, en esta investigación fue el postulado por Conway et al. (1994). Sus medidas de ajuste fueron las más pobres (ver tabla 3). En cuanto a la predicción de la formación de la FBM por parte del modelo, los dos caminos propuestos como determinantes directos (sorpresa y repetición) no fueron significativos para estos datos. En la figura 2, llama la atención que el camino que conecta el conocimiento previo con la atribución de importancia/consecuencia del evento tenga una significación negativa. Esto indica que cuanto más sabe la gente acerca de bin Laden, menos importancia le atribuye al evento. Una posible explicación para este hallazgo tendría que pensarse en términos de la cantidad de información que en noticieros de televisión y diarios impresos circuló desde el 11-S, alrededor de la figura de Osama bin Laden. Esto probablemente sea la base de un cúmulo de conocimiento, no necesariamente veraz, en torno a sus actividades en contra de los intereses americanos. La importancia de su muerte puede ser evaluada como importante para los EE.UU. y para sus ciudadanos, pero no tanto para una población como la colombiana, sometida a un conflicto armado durante más de 50 ańos, y a quien, probablemente, la muerte de un líder de un grupo terrorista internacional no le genera tanta expectativa y se valora con tanta importancia como a la de los propios fugitivos nacionales.

\section{Consideraciones para el contexto colombiano}

De acuerdo con la revisión bibliográfica de los autores, este es el primer trabajo latinoamericano en el que se estudian los determinantes para la formación de una FBM. Una crítica razonable es la elección de un hecho internacional como fuente investigativa, en lugar de elegir un 
hecho ocurrido en la región. Dos argumentos pueden esgrimirse como respaldo a la decisión de no incluir un evento ocurrido en Colombia. Por una parte, en el momento del desarrollo de la investigación, pocos eventos con el suficiente impacto como para desencadenar una FBM acontecieron en Colombia. Un punto fundamental es que los investigadores en FBM realizan un análisis rápido para tomar la decisión de cuál evento tiene una mayor probabilidad de alcanzar la suficiente fuerza como para transformarse en un hecho significativo que se relieve del flujo informativo cotidiano. En segundo lugar, los estudios en los que se han probado los diferentes modelos de formación de una FBM retoman eventos recientes. En este caso, se siguió la tradición investigativa en el campo de estudio.

Ahora bien, futuras investigaciones deberían considerar hechos relacionados con la historia reciente colombiana, con el ánimo de evaluar los modelos teóricos cuando la población del país está implicada, además para comprobar si las noticias políticas, o del conflicto en Colombia, tan repetitivas en los medios de comunicación, son susceptibles de convertirse en FBM. Esto ayudaría a comprender si parte de la historia del país se forma a través de recuerdos relacionadas con eventos políticos o armados, o bien la nueva historia de Colombia se edifica en recuerdos sobre eventos de corte menos violento, más ahora cuando las Fuerzas Armadas Revolucionarias de Colombia (FARC) firmaron el acuerdo para su desmovilización total. En efecto, luego de una larga negociación, Colombia dio por terminado el único conflicto armado interno activo en Latinoamérica. El tiempo que tome la implementación del acuerdo y la reinserción de los combatientes, incidirá en que este evento pueda ser considerado como poco sorpresivo por la población general, pero otras variables como la valoración de la importancia/ consecuencia del hecho, así como otras variables sociales y cognitivas tendrán que jugar un rol importante. En definitiva, una FBM pudo formarse y es entonces el momento propicio para indagar acerca de la red de FBM que pueden enlazarse y los mecanismos implicados en la formación de FBM para hechos valorados como muy positivos (Demiray \& Freund, 2015) para grupos específicos de la población 
(por ejemplo, las víctimas) o no tan positivos (aquel grupo poblacional a quien los acuerdos de paz no le parecieron benéficos).

Asimismo, es importante que se realice un corpus de recuerdos de hechos relacionados con el conflicto colombiano, el cual puede ser nutrido por diversas disciplinas. Luego de un conflicto tan prolongado, comprender los mecanismos psicológicos y sociales subyacentes a las secuelas de la guerra puede ser útil para el establecimiento de programas de intervención. No se debe olvidar que las FBM se pueden estudiar en grupos poblacionales específicos asumiéndose como memorias traumáticas (al respecto ver Van der Kolk \& Fisler, 1995), hecho que aportaría para la reconstrucción colectiva y la intervención en dichas poblaciones.

Por último, la metodología empleada para evaluar los modelos de FBM podría ser útil también para ser aplicada en otras áreas como la educación, marketing (Roehm, 2016) y el periodismo ya que estas disciplinas, entre otros objetivos, buscan crear memorias, posicionamientos, y crear comunicaciones que impacten socialmente. El análisis de qué eventos son los recordados de forma vívida por las personas ayudaría a entender el proceso de aprendizaje, a identificar qué elementos en una comunicación publicitaria crean una memoria a largo plazo y qué forma de presentar las noticias ayuda a retenerse en los oyentes.

Para culminar, este estudio presenta varias limitaciones. En primer lugar, el diseño no contempló una segunda aplicación del instrumento, como se realizó en el estudio original de Luminet \& Curci (2009), para calcular la estabilidad de los puntajes y la fiabilidad de la prueba. En segundo lugar, todos los participantes fueron voluntarios, no realizándose un muestreo probabilístico. En general, esta es una de las limitaciones comunes en muchos de los estudios de FBM, en parte debido a la rapidez con la cual los investigadores deben ajustar o crear las pruebas y aplicarlas. Futuros estudios deben propender por realizar seguimientos a corto, mediano y largo plazo, para explorar la precisión de los recuerdos, de acuerdo con el tipo de evento. 


\section{Referencias}

Bernsten, D. (2009). Flashbulb memories and social identity. En O. Luminet \& A. Curci. (Eds), Flashbulb memories. New Issues and New Perspectives. (pp. 187-205). Nueva York: Psychology Press.

Brown, R. \& Kulik, J. (1977). Flashbulb memories. Cognition, 5, 73-99. https://dx.doi.org/10.1016/0010-0277(77)90018-X

¿Cómo vive el hombre que mató a Osama Bin Laden? (2015, Febrero). Revista Semana. Recuperado de http://www.semana.com/gente/ articulo/como-vive-el-asesino-de-osama-bin-laden/417803-3

Conway, M., Anderson, S. J., Larsen, S. F., Donnelly, C. M., McDaniel. M. A., McClelland, A. G. R., Rawles, R. E. \& Logie, R. H. (1994). The formation of flashbulb memories. Memory \& Cognition, 22(3), 326-343. https://doi.org/10.3758/BF03200860

Curci, A. (2005). Latent variable models for the measurement of flashbulb memories: A comparative approach. Applied Cognitive Psychology, 19, 3-22. https://doi.org/10.1002/acp.1039

Curci, A. \& Luminet, O. (2009). Flashbulb Memories for Expected Events: A Test of the Emotional-Integrative Model. Applied Cognitive Psychology, 23, 98-114. https://doi.org/10.1002/acp. 1444

Curci, A. \& Luminet, O. (2006). Follow-up of a cross-national comparison on flashbulb and event memory for the September 11th attacks. Memory, 14, 329-344. https://doi.org/10.1002/ acp. 1444

Curci, A., Luminet, O., Finkenauer, C. \& Gisle, L. (2001). Flashbulb memories in social groups: A comparative test-retest study of the memory of French President Mitterrand's death in a French and Belgian group. Memory, 9, 81-101. https://doi.org/ $10.1080 / 09658210042000120$

Demiray, B. \& Freund, A. M. (2015). Michael Jackson, Bin Laden and I: Functions of positive and negative, public and private flashbulb memories. Memory, 23(4), 487-506. https://doi.org/1 $0.1080 / 09658211.2014 .907428$ 
Edery-Halpern, G. \& Nachson, I. (2004). Distinctiveness flashbulb memory: Comparative analysis of five terrorist attacks. Memory, 12 (2) 147-157. https://doi.org/10.1080/09658210244000432

Er, N. (2003). A New Flashbulb Memory Model Applied to the Marmara earthquake. Applied Cognitive Psychology, 17, 503-517. https://doi.org/10.1002/acp.870

Ferrando, P. J. \& Lorenzo-Seva, U. (2014). El análisis factorial exploratorio de los ítems: algunas consideraciones adicionales. Anales de Psicología, 30, 1170-1175. https://doi.org/10.6018/ analesps.30.3.199991

Finkenauer, C., Luminet, O., Gisle, L., El-Ahmadi, A., Van der Linden, M. \& Philippot, P. (1998). Flashbulb memories and the underlying mechanisms of their formation: Toward an emotional-integrative model. Memory \& Cognition, 26(3), 516531. https://doi.org/10.3758/BF03201160

Flament, C. \& Rouquette, M. L. (2003). Anatomie des idées ordinaries. Comment étudier les representations sociales. París: Armand Colin. Forigua-Rojas, E. (2010). Guerra en Afganistán: la experiencia soviética. Papel Político, 15, 183-234. Disponible en http://www. scielo.org.co/pdf/papel/v15n1/v15n1a08.pdf

Fridja, N. H. (1986). The emotions. Cambridge: Cambridge University Press.

Fridja, N. H., Kuipers, P. \& ter Schure, E. (1989). Relation among emotion, appraisal and emotional action readiness. Journal of Personality and Social Psychology 57, 212-228. https://doi. org/10.1037/0022-3514.57.2.212

Hair, J. F. Jr., Black, W. C., Babin, B. J. \& Anderson, R. E. (2010). Multivariate Data Analysis. Upper Saddle River: Pearson Prentice Hall.

Halbwachs, M. (2004). Los marcos sociales de la memoria. Barcelona: Anthropos.

Joscelyn, T. (2015, Marzo). Osama bin Laden's Files: Al Qaeda's deputy general manager in Yemen. FDD's Long War Journal. Recuperado de https://www.longwarjournal.org/archives/2015/03/ 
osama-bin-ladens-files-al-qaedas-deputy-general-manager-inyemen.php

Lanciano, T., Curci, A. \& Semin, G. (2010). The emotional and reconstructive determinants of emotional memories: An experimental approach to flashbulb memory investigation. Memory, 18, 473-475. doi: 10.1080/09658211003762076

Lanciano, T., Curci, A. \& Soleti, E. (2013). "I Knew It Would Happen... And I Remember It": The Flashbulb Memory for the Death of Pope John Paul II. Europe's Journal of Psychology, 9(2), 220-230. https://doi.org/10.1080/09658211003762076

Lazarus, R. \& Folkman, S. (1986). Estrés y procesos cognitivos. Barcelona: Martínez Roca.

Livingston, R. (1967). Reinforcement. En G. Quarton, T. Melenchunk \& F. Schmitt (Eds.). The neurosciences: A study program (pp. 514576). Nueva York: Rockfeller University Press.

Luminet, O. (2018). Using Structural Equation Modelling approaches to better understand the formation of Flashbulb memories. En: O. Luminet y A. Curci. (Eds). Flashbulb memories: New challenges and future perspectives. (pp. 48-72). Nueva York: Routledge.

Luminet, O. (2009). Models for the formation of flashbulb memories. En: O. Luminet y A. Curci. (Eds). Flashbulb memories. New Issues and New Perspectives. (pp. 51-73). Nueva York: Psychology Press.

Luminet, O. \& Curci, A. (2009). The 9/11 attacks inside and outside the US: Testing four models of flashbulb memory formation across groups and the specific effects of social identity. Memory, 17, 742-759. https://doi.org/10.1080/09658210903081827

Luminet, O., Curci, A., Marsh, E., Wessel, I., Constantin, T., Gencoz, F. \& Masao, Y. (2004). The Cognitive, Emotional and Social Impacts of the September 11 Attacks: Group Differences in Memory for the Reception Context and the Determinants of Flashbulb Memory. The Journal of General Psychology, 131, 197-224. https://doi.org/10.3200/GENP.131.3.197-224

Morse, C. K., Woodward, E. M. \& Zweigenhaft, R. L. (1993). Gender Differences in Flashbulb Memories Elicited by the Clarence 
Thomas Hearings. Journal of Social Psychology, 133, 453-458. https://doi.org/10.1080/00224545.1993.9712169

Neisser, U. (1982). Snapshots or benchmarks? En U. Neisser (Ed.), Memory observed: Remembering in natural context (pp. 43-48). San Francisco: W. H. Freeman.

Pérez, E., Medrano, L. A. \& Sánchez Rosas, J. (2013). El Path Analysis: conceptos básicos y ejemplos de aplicación. Revista Argentina de Ciencias del Comportamiento, 5(1), 52-66. Recuperado de http://revistas.unc.edu.ar/index.php/racc

Rateau, P. \& Lo Monaco, G. (2013). La Teoría de las Representaciones Sociales. Orientaciones conceptuales, campos de aplicaciones y métodos. Revista CES Psicología, 6, 22-42.

Roehm, M. L. (2016). An exploration of flashbulb memory. Journal of Consumer Psychology, 26(1), 1-16. https://doi.org/10.1016/j. jcps.2015.06.004

Ruiz, M., Pardo, A. \& San Martín, R. (2010). Modelos de ecuaciones estructurales. Papeles del Psicólogo, 31, 34-45. Recuperado de http://www.papelesdelpsicologo.es/pdf/1794.pdf

Tamayo Agudelo, W. (2012). Memorias Flashbulb y representaciones sociales. Propuesta para un estudio conjunto. Revista Psicoespacios, 6, 183-199.

Tamayo-Agudelo, W., Chaves Castaño, L. \& Cárdenas, J. (2017). Propiedades psicométricas de una prueba de Recuerdos Flashbulb. Revista Iberoamericana de Diagnóstico y Evaluación Psicológica, 45, 125-136. https://doi.org/10.21865/RIDEP45.3.10

Timmerman, M. E. \& Lorenzo-Seva, U. (2011). Dimensionality Assessment of Ordered Polytomous Items with Parallel Analysis. Psychological Methods, 16, 209-220. https://doi.org/10.1037/ a0023353

Tinti, C, Schmidt, S., Sotgiu, I., Testa, S. \& Curci, A. (2009). The Role of Importance/Consequentiality Appraisal in Flashbulb Memory Formation: The Case of the Death of Pope John Paul II. Applied Cognitive Psychology, 23, 236-253. https://doi.org/ 10.1002/acp. 1452 
Torres Vásquez, H. (2015). Conflicto armado y terrorismo en Colombia. El terrorismo de las Farc-EP de acuerdo con la jurisprudencia de la Corte Constitucional colombiana. Iustitia, 13, 11-34. Disponible en http://revistas.ustabuca.edu.co/index.php/IUSTITIA/ article/view/1535

Van der Kolk, B. A. \& Fisler, R. (1995). Dissociation and the fragmentary nature of traumatic memories: Overview and exploratory study. Journal of Traumatic Stress, 8(4), 505-525. https://doi. org/10.1002/jts.2490080402

Recibido: 25 de noviembre, 2016

Revisado: 23 de mayo, 2018

Aceptado: 11 de junio, 2018 